\title{
Comparação Estatística de Autovalores de um Operador de Schrödinger Perturbado
}

\author{
Statistical Comparison of Eigenvalues of a Disturbed Schrödinger Operator \\ Everton Artuso ${ }^{* 10}$, Cesar Marim ${ }^{1}$ \\ ${ }^{1}$ Universidade Federal da Fronteira Sul, Realeza, PR, Brasil
}

\begin{abstract}
Recebido em 23 de setembro de 2019. Revisado em 18 de janeiro de 2020. Aceito em 25 de janeiro de 2020
Nesse trabalho introduzimos os operadores de Schrödinger de forma ingênua e despretensiosa, mas munida de certa precisão e didática. São mencionados os tipos de potenciais mais estudados, os periódicos e os aleatórios e, via simulação numérica, comparados os autovalores da matriz truncada de um operador munido de um potencial periódico e com este mesmo potencial perturbado por uma variável aleatória contínua com distribuição uniforme. Avaliamos qual a magnitude necessária da variável aleatória para produzir diferença significativa entre os conjuntos de autovalores.
\end{abstract}

Palavras-chave: Operadores de Schrödinger, potencial periódico, potencial perturbado.

In this paper we introduce Schrödinger's operators in a naive and unpretentious way, but with some precision and didactics. The most studied periodic and random potential types are mentioned and, by numerical simulation, compare the eigenvalues of an operator's truncated matrix with a periodic potential and the same potential disturbed by an evenly distributed continuous random variable. We evaluated the magnitude of the random variable required to produce a significant difference between the eigenvalue sets.

Keywords: Schrödinger operators, periodic potential, disturbed potential.

\section{Introdução}

A física de matéria condensada de um elétron é uma simplificação que tem tido um sucesso notável na descrição de certas propriedades dos cristais. As noções básicas de materiais isolantes, condutores e semi-condutores podem ser explicadas pelo espectro de energia de um único elétron que se move sob a influência de um arranjo periódico de átomos e do princípio de exclusão de Pauli [1]. O último determina a ocupação dos estados por elétrons não interagentes. No modelo de um elétron, a interação entre os elétrons é negligenciada e as propriedades espectrais e de transporte do material são descritas pelo modelo de uma partícula do operador Hamiltoniano de Schrödinger.

De fato, em condutores e semi-condutores, elétrons livres têm que partilhar o espaço total disponível no interior do material - e por isso seus níveis de energia se empilham criando uma estrutura de bandas a partir de cada nível de energia atômico. Em bons condutores (metais) os elétrons estão tão fortemente degenerados que eles acabam por não contribuir de forma significativa para a capacidade térmica do metal. Muitas propriedades mecânicas, elétricas, magnéticas, ópticas e químicas dos sólidos são consequências diretas da repulsão de Pauli entre elétrons livres ou semi-livres. Para mais detalhes, veja [1] e [2].

Descrevendo a evolução temporal do elétron, a equação de Erwin Schrödinger dependente do tempo é dada por

$$
i \hbar \frac{\partial}{\partial t} \Psi(t, x)=-\frac{\hbar^{2}}{2 m} \Delta \Psi(t, x)+V(x) \Psi(t, x),
$$

onde $t$ é a variável temporal, $x$ é a variável espacial e $\hbar=\frac{h}{2 \pi}=1,05457 \times 10^{-34} \mathrm{Js}$ é a constante de Planck dividida por $2 \pi$. O operador Hamiltoniano $H$ será, por simplicidade tomando um sistema de unidades adequado para o qual $m=\frac{1}{2}$ e $\hbar=1$, escrito como

$$
H \Psi(t, x)=(-\Delta+V(x)) \Psi(t, x) .
$$

Ainda, é possível reescrever a equação (1) usando o operador $H$ como sendo $H \Psi=i \frac{\partial}{\partial t} \Psi$ para o qual o problema de existência e unicidade das soluções da equação (1) com o estado inicial $\Psi_{0}=\Psi(0, \cdot)$ dado pode ser reduzido ao problema do estabelecimento da auto-adjunticidade para o operador linear $H$, ou seja, se o operador for hermitiano está garantida a existência e unicidade de soluções com estado inicial $\Psi_{0}$.

A equação de Schrödinger (11) tem raízes no modelo ondulatório da mecânica quântica, começando da ideia

\footnotetext{
*Endereço de correspondência: everton.artuso@uffs.edu.br
} 
que uma partícula livre se comportaria como uma onda plana e poderia então ser descrita por uma função de onda $\Psi$ da forma

$$
\Psi(t, x)=A e^{i(k \cdot x-\omega t)},
$$

para todo $t \in \mathbb{R}$ e para todo $x \in \mathbb{R}^{d}$, a qual se propaga com velocidade constante $\frac{|v|}{2}=\frac{\omega}{|k|} \in(0, \infty)$ na direção $k \in \mathbb{R}^{d} \backslash\{0\}$. O $A$ da equação é um número complexo não nulo que representa a constante de normalização. Usando algumas das leis fundamentais da teoria quântica, a saber, a equação de Einstein

$$
E=\hbar \omega
$$

e a relação de Broglie para o comprimento de onda

$$
\lambda:=\frac{2 \pi}{k}=\frac{2 \pi \hbar}{m|v|}
$$

onde

$$
k=\frac{m|v|}{\hbar},
$$

chega-se a equação (1) quando se quer determinar a evolução temporal de $\Psi$ começando de algum estado inicial $\Psi_{0}$. Com efeito, a energia mecânica total $E$ de uma partícula de massa $m$ em termos da energia potencial $V(x)$, sendo $p$ o momentum da partícula, é

$$
E=\frac{p^{2}}{2 m}+V(x)
$$

Usando a equação (6), temos

$$
p=m|v|=\frac{m|v|}{\hbar} \hbar=k \hbar,
$$

ou seja, $p=k \hbar$, e pela equação (8), a equação (7) fica

$$
\hbar \omega=\frac{(\hbar k)^{2}}{2 m}+V(x) \text {. }
$$

Nos termos da equação (9), é particularmente difícil de escrever uma equação de onda para uma partícula por conta da energia potencial, por isso a ignoraremos por enquanto. Se a partícula tem massa nula, por exemplo um fóton, então a equação de onda eletromagnética seria apropriada, isto é, a equação de movimento é

$$
\frac{\partial^{2} \zeta(t, x)}{\partial x^{2}}=\frac{1}{c^{2}} \frac{\partial^{2} \zeta(t, x)}{\partial t^{2}}
$$

onde $\zeta(t, x)$ é a amplitude dependente do tempo e do espaço (campo elétrico ou magnético) da onda, e $c$ é a velocidade da luz. A solução da equação (10) é a onda plana

$$
\zeta(t, x)=A e^{i(k \cdot x-\omega t)},
$$

pois o movimento de onda sempre é descrito como função de $(x-|v| t)$, onde $|v|$ é a velocidade da onda, e $\omega=|v||k|$.
Para ondas eletromagnéticas, $|v|=c$, a velocidade da luz.

Aplicaremos a equação (10) ao caso de uma partícula material (massa não nula), mas, por simplicidade, continuemos com $V(x)=0$, caso denominado de "partícula livre". Substituindo a amplitude $\zeta(t, x)$ pela função de onda $\Psi(t, x)$, considerando $c \rightarrow v$ e assumindo uma solução tipo onda plana análoga a equação (11), inserimos esta solução na equação 10 , e após dividi-la por $\Psi(t, x)$ de ambos os lados da equação, obtemos

$$
(i k)^{2}=\frac{1}{v^{2}}(-i \omega)^{2}
$$

o que é inconsistente com a equação 9 pois, com $V(x)=0, k^{2} \propto \omega$ e não $\omega^{2}$. Para obter apenas a primeira potência de $\omega$, devemos derivar $\Psi(t, x)$ apenas uma vez com respeito ao tempo. Isto resulta na modificação da equação 10 , alterando $\zeta(t, x)$ por $\Psi(t, x)$ e $\frac{\partial^{2} \zeta(t, x)}{\partial t^{2}}$ por $\frac{\partial \Psi(t, x)}{\partial t}$, obtendo

$$
\frac{\partial^{2} \Psi(t, x)}{\partial x^{2}}=K \frac{\partial \Psi(t, x)}{\partial t}
$$

onde $K$ é uma constante. Inserindo a equação (11) na equação 13 e isolando $K$,

$$
\begin{aligned}
(i k)^{2} A e^{i(k \cdot x-\omega t)} & =\frac{\partial^{2} \Psi(t, x)}{\partial x^{2}} \\
& =K \frac{\partial \Psi(t, x)}{\partial t} \\
& =-i \omega K A e^{i(k \cdot x-\omega t)},
\end{aligned}
$$

que nos dá

$$
K=\frac{k^{2}}{i \omega} .
$$

Mas, da equação (9), com $V(x)=0$, temos que $\hbar \omega=\frac{\hbar^{2} k^{2}}{2 m}$ implica em $\frac{k^{2}}{\omega}=\frac{2 m}{\hbar}$, assim

$$
K=-i \frac{2 m}{\hbar}
$$

e portanto

$$
\frac{\partial^{2} \Psi(t, x)}{\partial x^{2}}=-i \frac{2 m}{\hbar} \frac{\partial \Psi(t, x)}{\partial t} .
$$

Multiplicando ambos os lados por $-\frac{\hbar^{2}}{2 m}$ temos

$$
-\frac{\hbar^{2}}{2 m} \frac{\partial^{2} \Psi(t, x)}{\partial x^{2}}=i \hbar \frac{\partial \Psi(t, x)}{\partial t} .
$$

Esta equação da onda é aplicável somente a uma partícula livre, isto é, uma partícula para a qual o comprimento de onda de de Broglie é constante por toda a parte. O comprimento de onda de de Broglie é constante pois a energia total é presumidamente constante, então a energia cinética, e portanto o momentum, são constantes. No caso de uma energia potencial constante, ainda é simples, 
pois o comprimento de onda de de Broglie é constante, e $V(x)=0$ é um caso particular de $V(x)=V_{0}=s$ constante. Portanto podemos, sem restrições, escrever

$$
\left(-\frac{\hbar^{2}}{2 m} \frac{\partial^{2}}{\partial x^{2}}+V_{0}\right) \Psi(t, x)=i \hbar \frac{\partial \Psi(t, x)}{\partial t},
$$

que é consistente com a equação $(9)$ com $V(x)$ no lugar de $V_{0}$. Em mecânica quântica, quantidades observáveis como a energia, momentum e posição são representadas por operadores. Não é um grande salto trocar em (21) $V_{0}$ por $V(x)$, para então obter a forma unidimensional da equação de Schrödinger dependente do tempo apresentada em 11,

$$
i \hbar \frac{\partial}{\partial t} \Psi(t, x)=\left(-\frac{\hbar^{2}}{2 m} \frac{\partial^{2}}{\partial x^{2}}+V(x)\right) \Psi(t, x) .
$$

Para mais detalhes, veja 3 .

Por simplicidade vamos considerar o exemplo em que $x \in \mathbb{R}$ dado por $\Psi(x)=B \operatorname{sen}(k \cdot x)+C \cos (k \cdot x)$, numa caixa rígida satisfazendo $\Psi(x)=0$ se $x<0$ ou $x>a$. Suponhamos também $V(x)=0$. Por continuidade, temos $\Psi(x=0)=\Psi(x=a)=0, \operatorname{logo} \Psi(x=0)=C=0 \mathrm{e}$ $\Psi(x=a)=B \operatorname{sen}(k \cdot a)=0$, de onde $k a=n \pi$, ou seja, $k_{n}=n \frac{\pi}{a}$ de onde $\lambda_{n}=\frac{2 \pi}{k_{n}}=\frac{2 a}{n}$, com $a=n \frac{\lambda}{2} \mathrm{e}$

$$
\Psi(x)=B \operatorname{sen}(k \cdot x) \text {. }
$$

Escrevendo $\Psi$ como uma série de Fourier, temos

$$
\Psi(t, x)=\sum_{n=0}^{\infty} c_{n} \Psi_{n}(t, x),
$$

com

$$
\Psi_{n}(t, x)=B \operatorname{sen}\left(k_{n} \cdot x\right) e^{-i \omega t} .
$$

$\operatorname{Como} \operatorname{sen}\left(k_{n} \cdot x\right)=\frac{e^{i k_{n} \cdot x}-e^{-i k_{n} \cdot x}}{2 i}$, temos

$$
\Psi_{n}(t, x)=\frac{B}{2 i}\left(e^{i\left(k_{n} \cdot x-\omega t\right)}-e^{-i\left(k_{n} \cdot x+\omega t\right)}\right) .
$$

Como $\Psi$ é uma função senoidal, sua segunda derivada também é uma função senoidal e satisfaz

$\frac{\partial^{2} \Psi(x)}{\partial x^{2}} \stackrel{23}{=} \frac{\partial^{2}(B \operatorname{sen}(k \cdot x))}{\partial x^{2}}=-k^{2} B \operatorname{sen}(k \cdot x)=-k^{2} \Psi(x)$, de onde

$$
\frac{\partial^{2} \Psi(x)}{\partial x^{2}}=-k^{2} \Psi(x) .
$$

Fazendo $V(x)=0$ em (7), por (4) e (9), temos

$$
E=K+V=\frac{p^{2}}{2 m}=\frac{\hbar^{2} k^{2}}{2 m},
$$

$\log O$

$$
k^{2}=\frac{2 m E}{\hbar^{2}},
$$

e como supomos $V(x)=0$, na verdade temos $E=K$, onde $K$ é a energia cinética, de onde, usando 30 , a equação 28 se torna

$$
\frac{\partial^{2} \Psi(x)}{\partial x^{2}}=-\frac{2 m K}{\hbar^{2}} \Psi(x) .
$$

Para um potencial $V(x)$ não nulo, temos que $K=E-V(x), \log \mathrm{O}$

$$
\frac{\partial^{2} \Psi(x)}{\partial x^{2}}=-\frac{2 m(E-V(x))}{\hbar^{2}} \Psi(x)
$$

nos dá

$$
\left(-\frac{\hbar^{2}}{2 m} \frac{\partial^{2}}{\partial x^{2}}+V(x)\right) \Psi(x)=E \Psi(x)
$$

chamada de equação de Schrödinger independente do tempo unidimensional que é obtida resolvendo a equação (1) por meio da separação de variáveis, como em [4].

Para o caso de maior dimensão, digamos $d$, o operador diferencial de segunda ordem dá lugar ao operador Laplaciano

$$
\Delta=\nabla^{2}=\sum_{i=1}^{d} \frac{\partial^{2}}{\partial x_{i}^{2}},
$$

e a equação de Schrödinger independente do tempo é escrita como

$$
\left(-\frac{\hbar^{2}}{2 m} \Delta+V(x)\right) \Psi(x)=E \Psi(x) .
$$

Escrevendo o operador Hamiltoniano como $H=-\frac{\hbar^{2}}{2 m} \Delta+V(x)$, então a equação 35 é de fato uma equação de autovalores (e autovetores) para $H$, que pode ser escrita de forma resumida simplesmente como

$$
H \Psi(x)=E \Psi(x) .
$$

\subsection{Resolvendo a equação de Schrödinger}

Utilizando o método de separação de variáveis, podemos escrever

$$
\Psi(t, x)=A e^{i(k \cdot x-\omega t)}=f(t) u(x),
$$

para $f(t)=\sqrt{A} e^{-i \omega t}$ e $u(x)=\sqrt{A} e^{i(k \cdot x)}$. Então, para $\left(t_{0}, x_{0}\right) \in \mathbb{R}^{1+d} \operatorname{com} \Psi\left(t_{0}, x_{0}\right) \neq 0$, temos

$$
\begin{aligned}
& f(t)=\frac{\sqrt{A} e^{-i \omega t} e^{i\left(k \cdot x_{0}\right)}}{e^{i\left(k \cdot x_{0}\right)}}=\frac{\Psi\left(t, x_{0}\right)}{\sqrt{A} e^{i\left(k \cdot x_{0}\right)}}=\frac{\Psi\left(t, x_{0}\right)}{u\left(x_{0}\right)}, \\
& u(x)=\frac{\sqrt{A} e^{i(k \cdot x)} e^{-i \omega t_{0}}}{e^{-i \omega t_{0}}}=\frac{\Psi\left(t_{0}, x\right)}{\sqrt{A} e^{-i \omega t_{0}}}=\frac{\Psi\left(t_{0}, x\right)}{f\left(t_{0}\right)}
\end{aligned}
$$


de onde $f \in \mathcal{C}^{1}(\mathbb{R})$ e $u \in \mathcal{C}^{2}\left(\mathbb{R}^{d}\right)$. Além disso, segue

$$
\frac{\partial}{\partial t} \Psi(t, x)=f^{\prime}(t) u(x)
$$

e

$$
\Delta \Psi(t, x)=\Delta(f(t) u(x))=f(t) \Delta u(x) .
$$

Avaliando em (2), temos

$$
\begin{aligned}
i f^{\prime}(t) u(x) & =(-\Delta+V(x)) \Psi(t, x) \\
& =-\Delta \Psi(t, x)+V(x) \Psi(t, x) \\
& =-f(t) \Delta u(x)+f(t) V(x) u(x) \\
& =f(t)(-\Delta+V(x)) u(x),
\end{aligned}
$$

e para $x=x_{0}$, tal que $u\left(x_{0}\right) \neq 0, f$ satisfaz a equação diferencial ordinária

(46)

$$
f^{\prime}(t)=i\left[\frac{\Delta u\left(x_{0}\right)-V\left(x_{0}\right) u\left(x_{0}\right)}{u\left(x_{0}\right)}\right] f(t)=-i \lambda f(t),
$$

cujas soluções gerais são do tipo $f(t)=c e^{-i \lambda t}$, para $c \in \mathbb{C} \backslash\{0\}$. Estas soluções gerais caracterizam completamente a função com relação ao tempo.

Como $i f^{\prime}(t) u(x)=f(t)(-\Delta+V(x)) u(x)$, ao substituirmos $f^{\prime}(t)$ obtemos

$$
i(-i \lambda f(t)) u(x)=f(t)(-\Delta+V(x)) u(x),
$$

de onde vem

$$
-\Delta u(x)+V(x) u(x)=\lambda u(x),
$$

para todo $x \in \mathbb{R}^{d}$, ou seja, a função $u$ satisfaz a equação de Schrödinger independente do tempo.

Seja $f: \mathbb{R} \rightarrow \mathbb{R}$. Dizemos que $f$ é limitada se existe algum $K \geq 0$ tal que $|f(t)| \leq K$, para todo $t \in \mathbb{R}$. Então, a evolução temporal dada por $f$ é limitada se, e só se, o autovalor $\lambda$ é real, uma vez que

$$
f(t)|=| c e^{-i \lambda t}|=| c e^{-i(\Re(\lambda)+i \Im(\lambda)) t}|=| c|| e^{\Im(\lambda) t} \mid,
$$

e $\left|e^{\Im(\lambda) t}\right|$ só é limitado se $\Im(\lambda)=0$. Essa é uma situação fisicamente relevante. Para um maior aprofundamento, consulte 4]. Vale mencionar que os autovalores são de fato reais, uma vez que o operador é auto-adjunto. Com efeito, considerando $H \psi=E \psi$, onde $\mathrm{H}$ é um operador Hamiltoniano autoadjunto (Hermitiano), $\log o H=H^{*}$, temos

$$
\langle H \psi, \phi\rangle=\left\langle\psi, H^{*} \phi\right\rangle=\langle\psi, H \phi\rangle .
$$

Por definição, temos

$$
\|\psi\|^{2}=\langle\psi, \psi\rangle=\int \overline{\psi(x)} \psi(x) d x
$$

então

$$
\begin{aligned}
E\|\psi\|^{2} & =E\langle\psi, \psi\rangle=\langle\psi, E \psi\rangle \\
& =\langle\psi, H \psi\rangle=\langle H \psi, \psi\rangle \\
& =\langle E \psi, \psi\rangle=\bar{E}\langle\psi, \psi\rangle=\bar{E}\|\psi\|^{2},
\end{aligned}
$$

assim $E=\bar{E}$, e portanto $E \in \mathbb{R}$. O número $E$ é o autovalor de $H$ associado a autofunção $\psi$.

\section{Operadores de Schrödinger Discretos}

Os operadores de Schrödinger discretos são definidos num espaço de sequências, a saber, o espaço de Hilbert

$$
\ell^{2}\left(\mathbb{Z}^{d}\right)=u: \mathbb{Z}^{d} \rightarrow \mathbb{C} ; \sum_{n \in \mathbb{Z}^{d}}|u(n)|^{2}<\infty,
$$

cuja norma é denotada por

$$
\|u\|=\left(\sum_{n \in \mathbb{Z}^{d}}|u(n)|^{2}\right)^{\frac{1}{2}} .
$$

Quando uma partícula se move no reticulado $\mathbb{Z}^{d}$, supondo que $\|u\|=1$, a probabilidade de encontrarmos tal partícula no ponto $n \in \mathbb{Z}^{d}$ é dada por $|u(n)|^{2}$, onde $u(n)$ é uma sequência indexada por $\mathbb{Z}^{d}$. É conveniente equipar $\mathbb{Z}^{d}$ com a norma

$$
\|n\|_{1}=\sum_{\nu=1}^{d}\left|n_{\nu}\right|
$$

Esta norma reflete a estrutura de reticulado de $\mathbb{Z}^{d}$. Dois vértices $n$ e $m$ de $\mathbb{Z}^{d}$ são conectados por uma aresta, se eles forem vizinhos próximos, ou seja, se $\|n-m\|_{1}=1$. Para $n, m \in \mathbb{Z}^{d}$ a norma $\|n-m\|_{1}$ dá o comprimento do menor caminho, não necessariamente único, entre $n$ e $m$. $\mathrm{O}$ operador $H_{0}$, a energia cinética, é o análogo discreto de $-\Delta$, a saber

$$
H_{0}(u(n))=-\sum_{\|m-n\|_{1}=1}(u(m)-u(n)) .
$$

Este operador é chamado de Laplaciano discreto para o reticulado $\mathbb{Z}^{d}$. Sua representação matricial é dada por

$$
H_{0}(i, j)=\left\{\begin{array}{cl}
-1 & \text { se }\|i-j\|_{1}=1 \\
2 d & \text { se } i=j, \\
0 & \text { caso contrário }
\end{array}\right.
$$


ou seja,

$$
H_{0}=\left(\begin{array}{ccccccc}
\ddots & \ddots & \ddots & \ddots & & & \\
\ddots & -1 & 2 & -1 & 0 & & \\
& 0 & -1 & 2 & -1 & 0 & \\
& & 0 & -1 & 2 & -1 & \ddots \\
& & & \ddots & \ddots & \ddots & \ddots
\end{array}\right)
$$

para o caso unidimensional. Por simplicidade, consideramos $H_{0}^{\prime}=H_{0}-2 d I$, assim o operador de Schrödinger discreto unidimensional é dado por $H=-H_{0}^{\prime}+V$ na matriz 62):

$$
\left(\begin{array}{ccccccc}
\ddots & \ddots & \ddots & \ddots & & & \\
\ddots & 1 & V_{(n-1)} & 1 & 0 & & \\
& 0 & 1 & V_{(n)} & 1 & 0 & \\
& & 0 & 1 & V_{(n+1)} & 1 & \ddots \\
& & & \ddots & \ddots & \ddots & \ddots
\end{array}\right) .
$$

A equação de Schrödinger independente do tempo, $H \psi=E \psi$, toma a forma matricial (63):

$$
\left(\begin{array}{ccccccc}
\ddots & \ddots & \ddots & \ddots & & & \\
\ddots & 1 & V_{(n-1)} & 1 & 0 & & \\
& 0 & 1 & V_{(n)} & 1 & 0 & \\
& & 0 & 1 & V_{(n+1)} & 1 & \ddots \\
& & & \ddots & \ddots & \ddots & \ddots
\end{array}\right)\left(\begin{array}{c}
\vdots \\
\psi_{(n-1)} \\
\psi_{(n)} \\
\psi_{(n+1)} \\
\vdots
\end{array}\right)=E\left(\begin{array}{c}
\vdots \\
\psi_{(n-1)} \\
\psi_{(n)} \\
\psi_{(n+1)} \\
\vdots
\end{array}\right) .
$$

O operador é representado pela equação de recorrência, para $n \in \mathbb{Z}$ e $\psi \in \ell^{2}(\mathbb{Z})$, dada por

$$
[H \psi]_{(n)}=\psi_{(n-1)}+V_{(n)} \psi_{(n)}+\psi_{(n+1)},
$$

cuja equação de autovalores e autoestados é dada por

$$
\psi_{(n-1)}+V_{(n)} \psi_{(n)}+\psi_{(n+1)}=E \psi_{(n)} .
$$

\section{Potenciais}

Considere o potencial periódico $V(x)$ em uma dimensão satisfazendo $V(x+n a)=V(x)$, para $x \in \mathbb{R}$, onde $a$ é a constante da rede discreta e $n$ é um inteiro. A equação de Schrödinger para autovalores de energia para um elétron é dada por

$$
(-\Delta+V(x)) \psi_{\lambda}(x)=\lambda \psi_{\lambda}(x) .
$$

Claramente, a função de onda $\psi_{\lambda}(x+n a)$ também satisfaz a mesma equação. Assim, $\psi_{\lambda}(x+n a)$ se diferencia de $\psi_{\lambda}(x)$ somente por uma fase independente de $x$, ou seja,

$$
\psi_{\lambda}(x+n a)=e^{i k n a} \psi_{\lambda}(x)
$$

onde $k$ é um número real. A equação (67) representa, em uma de suas formas, o Teorema de Bloch [5], a qual gera importantes consequências na teoria de condução de elétrons. Considerando que $\psi(x)$ e $\psi(x+n a)$ satisfazem a mesma equação, elas são linearmente dependentes, ou seja,

$$
\psi(x+n a)=c(n a) \psi(x) .
$$

Usando o mesmo argumento outra vez, obtemos

$$
\begin{aligned}
\psi(x+n a+m a) & =c(n a) \psi(x+m a) \\
& =c(n a) c(m a) \psi(x) \\
& 68(n a+m a) \psi(x) .
\end{aligned}
$$

Como a função de onda $(\psi(x))$ não se anula em geral, obtemos $c(n a+m a)=c(n a) c(m a)$, ou seja,

$$
c(x+y)=c(x) c(y),
$$

conhecida como equação funcional exponencial de Cauchy.

Lema 3.1 A equação funcional exponencial de Cauchy $c(x+y)=c(x) c(y)$ tem por solução geral $c(y)=e^{A(x)}$, onde $A: \mathbb{R} \rightarrow \mathbb{R}$ é uma função aditiva, ou $c(y)=0$.

Demonstração. De fato, $c(y)=0$ é solução da equação (69), para todo $y \in \mathbb{R}$. Suponha que $c\left(y_{0}\right)=0$ para algum $y_{0}$. Logo,

$$
c(y)=c\left(\left(y-y_{0}\right)+y_{0}\right)=c\left(y-y_{0}\right) c\left(y_{0}\right)=0
$$

para todo $y \in \mathbb{R}$, então se a função $c$ se anula num ponto, ela é identicamente nula. Note que $c(x)=e^{x}$ satisfaz a 
equação 69, logo esta admite solução não trivial. Além disso, a função $c(y)$ é estritamente positiva. Com efeito, tome por exemplo $y=\frac{t}{2}$ na equação $(69)$, logo teremos

$$
c(t)=c\left(\frac{t}{2}+\frac{t}{2}\right)=c\left(\frac{t}{2}\right) c\left(\frac{t}{2}\right)=\left[c\left(\frac{t}{2}\right)\right]^{2}>0 .
$$

Tomando o logaritmo natural em ambos os membros de (69), obtemos $\log c(x+y)=\log c(x)+\log c(y)$, e chamando $A(y)=\log c(y)$, verificamos que

$$
A(x+y)=A(x)+A(y),
$$

ou seja, a função $A$ é uma função aditiva. Além disso, $c(y)=e^{A(y)}$ é uma solução geral para a equação 69 , o que demonstra o resultado.

Em particular, $c(y)=e^{\theta y}$ é uma solução da equação (69), onde $\theta$ é uma constante. Como a função $\psi$ na equação (67) deve ser finita para todos os intervalos, a constante $\theta$ deve ser um número imaginário puro $\theta=i k$, onde $k$ é um número real, logo

$$
c(y)=e^{i k y} .
$$

Combinando as equações 68 com 73 , obtemos

$$
\psi_{\lambda}(x+n a)=e^{i k n a} \psi_{\lambda}(x)
$$

o que mostra o Teorema 3.2 seguinte:

Teorema 3.2 (de Bloch) As soluções da equação de Schrödinguer

$$
(-\Delta+V(x)) \psi_{\lambda}(x)=\lambda \psi_{\lambda}(x)
$$

para um potencial periódico $V(x)$ satisfazem

$$
\psi_{\lambda}(x+r)=e^{i k r} \psi_{\lambda}(x)
$$

onde $r$ é o período da função de onda $\psi_{\lambda}$.

Para mais detalhes, veja [6] e 7].

Nos cristais, os átomos ou os núcleos são distribuídos em uma rede periódica (digamos a rede $\mathbb{Z}^{d}$ por simplicidade) de uma maneira completamente regular. Vamos assumir que uma partícula (elétron) no ponto $x \in \mathbb{R}^{d}$ é estimulada por um potencial da forma $q f(x-i)$ devido a um átomo (ou íon, ou núcleo) localizado no ponto $i \in \mathbb{Z}^{d}$. Aqui, a constante $q$ (carga ou constante de acoplamento, em termos físicos) poderia ser absorvida na função $f$. No entanto, uma vez que vamos variar essa quantidade de átomo para átomo mais tarde, é útil escrever o potencial da forma acima. Então, em um cristal regular, nossa partícula está exposta a um potencial total

$$
V(x)=\sum_{i \in \mathbb{Z}^{d}} q f(x-i) .
$$

A função $f$ é chamada de potencial de um único sítio para distinguir do potencial total $V$. O potencial $V$ em
(77) é periódico com respeito ao reticulado $\mathbb{Z}^{d}$, ou seja, $V(x-i)=V(x)$ para todo $x \in \mathbb{R}^{d}$ e $i \in \mathbb{Z}^{d}$. A teoria matemática dos operadores de Schrödinger com potenciais periódicos está bem desenvolvida e baseia-se em uma análise minuciosa das propriedades de simetria dos operadores periódicos. Por exemplo, sabe-se que tais operadores apresentam, em geral, um espectro com estrutura de banda, isto é, para $a_{n}<b_{n}<a_{n+1}$,

$$
\sigma(H)=\bigcup_{n=0}^{\infty}\left[a_{n}, b_{n}\right]
$$

Este espectro também é conhecido como sendo espectro absolutamente contínuo.

Nas últimas décadas, os operadores de Schrödinger aleatórios, que se originaram na física da matéria condensada, foram estudados intensamente e de forma muito produtiva. A teoria está na interface de uma série de campos matemáticos: a teoria dos operadores, as equações diferenciais parciais, a teoria das probabilidades, em particular o estudo de processos estocásticos, o de passeios aleatórios e o movimento browniano em um ambiente aleatório. Os operadores de Schrödinger aleatórios modelam sólidos desordenados. Os sólidos aparecem na natureza de várias formas, sendo que em alguns casos são (quase) totalmente ordenados.

A maioria dos sólidos não constitui um cristal ideal. As posições dos átomos podem se desviar das posições de rede ideais de maneira não regular devido a imperfeições no processo de cristalização, ou as posições dos átomos podem estar completamente desordenadas, como é o caso em materiais amorfos ou vítreos. O sólido também pode ser uma mistura de vários materiais, como é o caso, por exemplo, de ligas ou semicondutores dopados (com impurezas intencionalmente introduzidas com propósito de modificar suas propriedades elétricas). Em todos esses casos, parece razoável considerar o potencial como uma quantidade aleatória. Por exemplo, se o material for puro, mas as posições dos átomos se desviam das posições da rede ideais aleatoriamente, podemos considerar um potencial aleatório da forma

$$
V_{\omega}(x)=\sum_{i \in \mathbb{Z}^{d}} q f\left(x-i-\xi_{i}(\omega)\right) .
$$

Aqui, $\xi_{i}$ são variáveis aleatórias que descrevem o desvio do $i$-ésimo átomo da posição $i$ da rede. Pode-se, por exemplo, assumir que as variáveis aleatórias são independentes e identicamente distribuídas. O sub-índice $\omega$ do potencial $V$ aparece para deixar claro que $V_{\omega}$ depende dos parâmetros aleatórios (desconhecidos). Para modelar um material amorfo como vidro ou borracha, assumimos que os átomos do material estão localizados em pontos $\omega_{i}$ completamente aleatórios no espaço. Esse potencial aleatório pode ser formalmente escrito como

$$
V_{\omega}(x)=\sum_{i \in \mathbb{Z}^{d}} q f\left(x-\omega_{i}(\omega)\right)
$$


Para mais detalhes, veja 8 .

\section{Metodologia}

Realizamos simulações no software Mathematica ${ }^{\circledR}$, para um caso bastante simplificado, encontrando o espectro (conjunto de autovalores, na verdade) do operador periódico cujo potencial foi dado pela tal função. Acoplamos ao potencial uma variável aleatória a fim de tornar o potencial periódico aleatório e executamos simulações computacionais para comparar estatisticamente os resultados do espectro do operador de Schrödinger com cada um dos potenciais. Primeiramente simulamos usando uma matriz truncada do operador com potencial periódico, que usamos para comparar com todos os outros. Após encontrarmos diferenças significativas entre o espectro do operador periódico e do perturbado, realizamos 20 simulações similares para efeito de amostragem.

\section{Resultados}

Conforme 8], em sistemas unidimensinais, um distúrbio arbitrariamente pequeno em um operador com potencial periódico, tornando-o levemente desordenado, mudará o espectro total de absolutamente contínuo (composto de bandas) para puramente pontual e, portanto, de condutor para isolante.

Vamos considerar um "caminho"unidimensional na rede bidimensional. Para investigar nosso caso, consideramos o potencial

$$
V(x, y)=10 \sum_{m, n \in \mathbb{Z}} \exp \left(-\frac{(x-m)^{2}+(y-n)^{2}}{0,00724}\right),
$$

$\begin{array}{ccccccccc}7,0800 & 1 & 0 & 0 & 0 & 0 & 0 & 0 & 0 \\ 1 & 2,5127 & 1 & 0 & 0 & 0 & 0 & 0 & 0 \\ 0 & 1 & 0,4470 & 1 & 0 & 0 & 0 & 0 & 0 \\ 0 & 0 & 1 & 0,0398 & 1 & 0 & 0 & 0 & 0 \\ 0 & 0 & 0 & 1 & 0,0017 & 1 & 0 & 0 & 0 \\ 0 & 0 & 0 & 0 & 1 & 3,99 \times 10^{-5} & 1 & 0 & 0 \\ 0 & 0 & 0 & 0 & 0 & 1 & . & 1 & 0 \\ 0 & 0 & 0 & 0 & 0 & 0 & 1 & . & 1 \\ 0 & 0 & 0 & 0 & 0 & 0 & 0 & 1 & \\ 0 & 0 & 0 & 0 & 0 & 0 & 0 & 0 & 1 \\ 0 & 0 & 0 & 0 & 0 & 0 & 0 & 0 & 0 \\ 0 & 0 & 0 & 0 & 0 & 0 & 0 & 0 & 0 \\ 0 & 0 & 0 & 0 & 0 & 0 & 0 & 0 & 0 \\ 0 & 0 & 0 & 0 & 0 & 0 & 0 & 0 & 0 \\ 0 & 0 & 0 & 0 & 0 & 0 & 0 & 0 & 0 \\ 0 & 0 & 0 & 0 & 0 & 0 & 0 & 0 & 0 \\ 0 & 0 & 0 & 0 & 0 & 0 & 0 & 0 & 0 \\ 0 & 0 & 0 & 0 & 0 & 0 & 0 & 0 & 0 \\ 0 & 0 & 0 & 0 & 0 & 0 & 0 & 0 & 0 \\ 0 & 0 & 0 & 0 & 0 & 0 & 0 & 0 & 0\end{array}$

(83)

Na Figura 1, temos o conjunto dos autovalores no plano complexo. Embora com 201 valores, o espectro está longe de ser normalmente distribuído, uma vez que o teste de normalidade de Shapiro-Wilk nos deu um $p$ valor de $4,59595 \times 10^{-15}$, o que é bem menor que os onde o denominador $2 \sigma^{2}=0,00724$, que é o dobro da variância, foi calculado de modo a ajustar o período da função, $(x, y) \in \mathbb{R}^{2}$, e o fator 10 que multiplica a série serve simplesmente para facilitar a visualização dos autovalores na simulação. De fato, a função $V$ está bem definida para todo $(x, y) \in \mathbb{R}^{2}$, um vez que

$$
\begin{aligned}
& V(x, y)=10 \sum_{m, n \in \mathbb{Z}} \exp \left(-\frac{(x-m)^{2}+(y-n)^{2}}{0,00724}\right) \\
& \leq 10 \iint_{\mathbb{R}^{2}} \exp \left(-\frac{(x-u)^{2}+(y-v)^{2}}{0,00724}\right) d u d v \\
& \quad+40<+\infty
\end{aligned}
$$

e a integral da distribuição Gaussiana converge. O 40 compensa o valor no qual a série é superior a integral numa vizinhança de $(x, y)=(0,0)$.

Para fins de simulação, truncamos a matriz (bi-infinita) (Equação (62) ) do operador em uma matriz de $M_{201} \mathrm{com}$ 201 linhas e 201 colunas, sendo que o valor 201 foi escolhido pois tomamos $-100 \leq m \leq 100$ e $-100 \leq n \leq 100$. Assim, o potencial fica dado por

$$
\begin{aligned}
& V(x, y)= \\
& 10 \sum_{m=-100}^{100} \sum_{n=-100}^{100} \exp \left(-\frac{(x-m)^{2}+(y-n)^{2}}{0,00724}\right),
\end{aligned}
$$

e o período da função foi dividido em 20 partes, de modo a facilitar a visualização. O primeiro bloco da matriz, com 20 linhas e 20 colunas (com valores arredondados) é dado pela matriz 83 :
0, 05 costumeiros, para um intervalo de $95 \%$ de confiança. Para avaliarmos o espectro com "impurezas"induzidas pela aleatoriedade, considere as variáveis aleatórias $\xi_{1} \mathrm{e}$ $\xi_{2} \operatorname{com} \xi_{j} \in[0,1]$ e distribuição uniforme, e o vetor aleatório $\xi=\left(\xi_{1}, \xi_{2}\right)$. Uma vez que estamos lidando com o 
truncamento da matriz do operador, não poderemos perceber diferenças significativas simplesmente comparando o retrato de cada espectro. Com propósito de comparar se há diferenças significativas entre os dois espectros, utilizamos um teste de hipóteses. Para o potencial com perturbação $10 \xi$,

$$
V_{10 \xi}(x, y)=10 \sum_{m=-100}^{100} \sum_{n=-100}^{100} \exp \left(-\frac{\left(\left(1-10 \xi_{1}\right) x-m\right)^{2}+\left(\left(1-10 \xi_{2}\right) y-n\right)^{2}}{0,00724}\right) .
$$

O espectro do operador com potencial perturbado (com fator $10 \xi)$ no plano complexo é apresentado na Figura 2 . O espectro não é normalmente distribuído, com $p$-valor de $1,295 \times 10^{-10}$ no teste de Shapiro-Wilk.

Utilizando o teste de hipóteses para dados nãoparamétricos de Mann-Whitney, obtemos um $p$-valor médio de 0,073495 , que é pouco maior que 0,05 para um intervalo de $95 \%$ de confiança, quando comparado com o espectro do operador periódico, logo os espectros não são significativamente diferentes. Realizamos 20 simulações para este caso, as quais são apresentadas na Tabela 1.

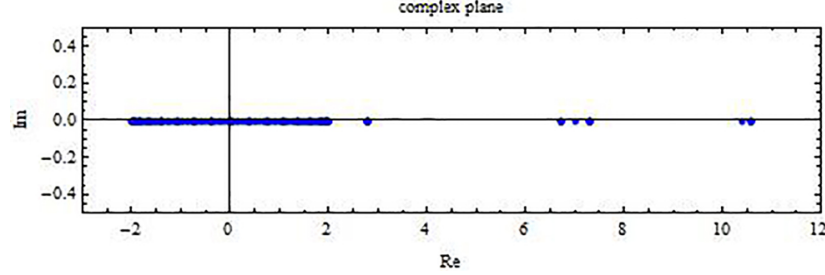

Figura 1: Espectro do operador com potencial periódico $V(x, y)$ no plano complexo.

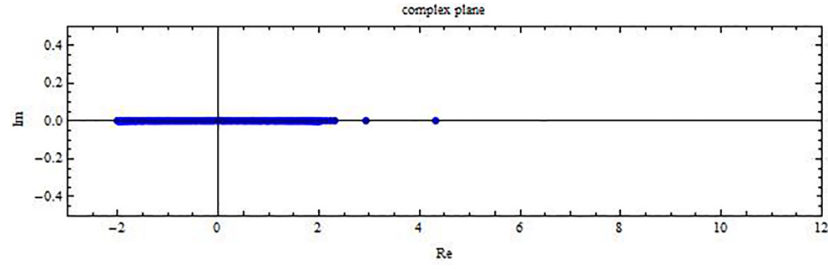

Figura 2: Espectro do operador com potencial perturbado $V_{10 \xi}(x, y)$ no plano complexo.

Finalmente, para o potencial com perturbação $10^{2} \xi$,

$$
V_{10^{2} \xi}(x, y)=10 \sum_{m=-100}^{100} \sum_{n=-100}^{100} \exp \left(-\frac{\left(\left(1-100 \xi_{1}\right) x-m\right)^{2}+\left(\left(1-100 \xi_{2}\right) y-n\right)^{2}}{0,00724}\right) .
$$

O espectro do operador com potencial perturbado (com fator $10^{2} \xi$ ) no plano complexo é apresentado na Figura 3 . O espectro não é normalmente distribuído, com $p$-valor de $2,3045 \times 10^{-10}$ no teste de Shapiro-Wilk. Utilizando o teste de hipóteses para dados não-paramétricos de MannWhitney, obtemos um $p$-valor médio de 0,03579 , que é pouco menor que 0,05 para um intervalo de $95 \%$ de confiança, quando comparado com o espectro do operador periódico, logo os espectros, nesse caso, são significativamente diferentes. Realizamos 20 simulações para este caso, as quais são apresentadas na Tabela 2.

\section{Considerações Finais}

Fomos capazes de perceber diferenças significativas entre o espectro do operador com potencial periódico e do perturbado, todavia precisamos de uma perturbação da ordem de $10^{2} \xi$, com $\xi \in[0,1]^{2}$ uniformemente distribuída.
Para os valores de perturbações menores testados, não podemos distinguir estatisticamente os dois espectros. Para mais informações sobre programação em Mathematica, veja 9 .

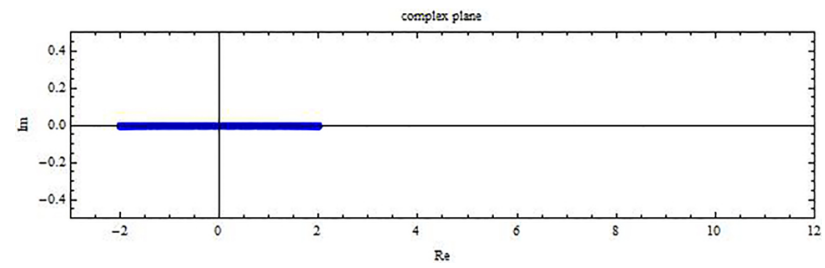

Figura 3: Espectro do operador com potencial perturbado $V_{10^{2} \xi}(x, y)$ no plano complexo. 
Tabela 1: $p$-valores para o fator $10 \xi$.

\begin{tabular}{cccccccc}
\hline Simulação & 1 & 2 & 3 & 4 & 5 & 6 & 7 \\
\hline$p$-valor & 0,0687 & 0,0615 & 0,0836 & 0,0875 & 0,0589 & 0,0655 & 0,0782 \\
Simulação & 8 & 9 & 10 & 11 & 12 & 13 & 14 \\
$p$-valor & 0,0606 & 0,0622 & 0,1204 & 0,0475 & 0,0985 & 0,0679 & 0,0729 \\
Simulação & 15 & 16 & 17 & 18 & 19 & 20 & \\
$p$-valor & 0,0580 & 0,0726 & 0,0550 & 0,0469 & 0,1091 & 0,0944 & \\
\hline
\end{tabular}

Tabela 2: $p$-valores para o fator $10^{2} \xi$.

\begin{tabular}{cccccccc}
\hline Simulação & 1 & 2 & 3 & 4 & 5 & 6 & 7 \\
$p$-valor & 0,0314 & 0,0335 & 0,0314 & 0,0365 & 0,0338 & 0,0314 & 0,0395 \\
Simulação & 8 & 9 & 10 & 11 & 12 & 13 & 14 \\
$p$-valor & 0,0392 & 0,0391 & 0,0314 & 0,0388 & 0,0466 & 0,0409 & 0,0346 \\
Simulação & 15 & 16 & 17 & 18 & 19 & 20 & \\
$p$-valor & 0,0317 & 0,0319 & 0,0316 & 0,0441 & 0,0361 & 0,0323 & \\
\hline
\end{tabular}

\section{Agradecimentos}

Os autores agradecem ao professor Leonardo de Lima pela revisão minuciosa de parte deste trabalho e pelo incentivo; e ao professor Tobias Heimfarth pelas conversas sobre a implementação computacional.

\section{Referências}

[1] M. Massimi, Pauli's Exclusion Principle: The Origin and Validation of a Scientific Principle (Cambridge University Press, Cambridge, 2005).

[2] D.J. Griffiths, Introduction to Quantum Mechanics (Prentice Hall, New Jersey, 2004), $2^{\text {a }}$ ed.
[3] C.E. Burkhardt e J.J. Leventhal, Foundations of Quantum Physics (Springer, New York, 2008).

[4] A.M. Hinz, em: Spectral Theory of Schrödinger Operators, editado por R. del Rio e C. Villegas-Blas (American Mathematical Society, Rhode Island, 2004).

[5] F. Bloch, Zeitschrift für Physik 52, 555 (1929).

[6] S. Fujita e K. Ito, Quantum Theory of Conducting Matter (Springer, New York, 2007).

[7] C. Kittel, Introduction to Solid State Physics (John Wiley and Sons, New Jersey, 1996), $7^{\mathrm{a}}$ ed.

[8] W. Kirsch, in: Random Schrödinger operators (Société Mathématique de France, Paris, 2008), v. 25, p. 1.

[9] P.R. Wellin, R.J. Gaylord e S.N. Kamin, An Introduction to Programming with Mathematica (Cambridge University Press, New York, 2005), $3^{\text {a }}$ ed. 